\title{
Overcoming barriers impeding early access of children and adolescents with cancer to emerging new therapeutic platforms and precision medicines
}

\author{
Fatih M Uckun* and Nicholas Kenny \\ Syneos Health, Global Oncology and Hematology Program \& Oncology Center of Excellence, USA
}

\begin{abstract}
Childhood cancer remains the leading cause of disease-related death of children in the United States (US) despite advances in survival rates for some types of childhood cancer. One in 5 children diagnosed with cancer in the US will not survive their disease, and of those who do, 2/3rds of survivors will suffer at least one treatment-related chronic disease. Because many childhood cancers are relatively rare, biotechnology firms and pharmaceutical companies have long argued that developing drugs specifically in a childhood cancer setting is not economically viable; the market is too small to justify the enormous expense. Instead, in many instances, drugs are developed in an adult setting and then "retrofitted" to the childhood setting - with all of the attendant concerns this brings. So, for example, this can lead to significant delays in the evaluation and availability of promising new therapies in poor prognosis pediatric cancer patients who are in urgent need for therapeutic innovations [1-14] - despite the fact that many childhood cancers share much in common with those in adults. There is growing consensus among pediatric hematologists-oncologists, US Food and Drug Administration (FDA), European Medicines Agency (EMA), coalitions of subject matter experts, support groups and other stakeholders that action is needed to remove such barriers that contribute to the unsatisfactory progress in improving the survival outcomes of adolescents with cancer $[3,10,12,13,15,16]$. In short, how can we better assure access for children /adolescents to disease and target-appropriate adult oncology clinical trials?
\end{abstract}

\section{Current Initiatives}

Recently, there has been a major effort to initiate early phase trials of targeted therapies in children and adolescents with cancer. For example, umbrella trial initiatives aimed at allowing children and adolescents with relapsed or refractory pediatric cancers early access to promising targeted precision medicines. The NCT03155620 Children's Oncology Group (COG)-NCI Pediatric MATCH Screening Trial is one example. The European Proof-of-Concept Therapeutic Stratification Trial of Molecular Anomalies in Relapsed or Refractory Tumors (ESMART) (NCT02813135) - which is part of the Innovative Therapies for Children with Cancer Precision Cancer Medicine Program, a European academic consortium [17], also provides an unique opportunity for new therapeutic innovations in pediatric hematologyoncology through mechanistic hypothesis-driven studies. Lessons and insights learned from these trials are likely to provide the foundation for effective and personalized precision medicine strategies for children and adolescents with difficult-to-treat cancers. For example, durable objective responses were observed in pediatric patients with anaplastic large-cell lymphoma and inflammatory myofibroblasic tumors harboring ALK fusions who were treated in a Phase I/II setting with the ALK inhibitor crizotinib [17]. Likewise, the Phase 1 testing of the TRK inhibitor larotrectinib for TRK fusion positive malignancies generated an exciting objective response rate of $>90 \%$ [18].

In parallel to these trial initiatives, there is ongoing regulatory dialog and emergent guidance. Both FDA and EMA have launched new regulatory initiatives aimed at improving the access of pediatric cancer patients to novel therapies developed for adults with cancer.
The European Pediatric Medicine Regulation [(EC)-No1901/2006)] mandated the establishment of the EMA's Pediatric Committee to provide guidance to pharmaceutical companies regarding their Pediatric Investigation Plans (PIPs) for active investigational drugs [9]. The multi-stakeholder platform ACCELERATE (http://www. accelerate-platform.eu) presented a consensus expert opinion in support of early drug access for adolescents with cancer - indicating that enrollment of adolescents of 12 years and over in adult early-phase clinical drug trials would represent a safe and efficient strategy in drug development [7]. Several changes were proposed by ACCELERATE to facilitate adolescents' access to early drug development programs, including that (i) there should be no set upper or lower age limit criteria for Phase II and Phase III trials for cancers that are present in both pediatric and adult populations with similar biology and (ii) adolescents over 12 years of age should be included from the onset of the cancer drug development process in adults [7].

In June 2018, FDA issued a Draft Guidance entitled "Considerations for the Inclusion of Adolescent Patients in Adult Oncology Clinical Trials" [5] emphasizing that pediatric oncology drug development should be coordinated with oncology drug development for adults as part of an overall drug development plan. The draft Guidance details a series of recommendations regarding inclusion of pediatric

*Correspondence to: Fatih Uckun, MD, PhD, Executive Medical Director, Syneos Health, 3201 Beechleaf Court, Suite 600, Raleigh, NC 27604, USA E-mail: fatih.uckun@att.net

Received: August 24, 2018; Accepted: September 24, 2018; Published: September 28, 2018 
patients in adult oncology trials in the US, and is based on a previous FDA publication [3]; this can be viewed as a strong endorsement of the recent ACCELERATE proposal [7] in Europe. The new FDA recommendations would certainly expand the options available for adolescent cancer patients who have relapsed after, or are refractory to, standard therapeutic strategies with no curative options, or for whom no standard therapies with curative intent exist. The Draft Guidance suggests that adolescent patients may be enrolled in first-in-human clinical trials after initial adult PK and toxicity data are obtained - a measure aimed at providing appropriate risk mitigation for adolescents [5]. Furthermore, the important provisions of the Race for Children Act, which is incorporated as Title $\mathrm{V}$ of the FDA Reauthorization Act (FDARA) that was enacted on August 18, 2017 (FD\&C Act Sec. 505B (a)(3), 21 USC 355c (a)(3), Public Law 115-52) has created a mechanism to expedite the evaluation of novel medicines with the potential to address the unmet need in the pediatric population by requiring pediatric investigation of appropriate new drugs intended for adults with cancer [19]. Specifically, Title V requires evaluation of new molecularly targeted drugs and biologics "intended for the treatment of adult cancers and directed at a molecular target substantially relevant to the growth or progression of a pediatric cancer" in molecularly targeted pediatric cancer investigation to generate clinically meaningful study data, "using appropriate formulations, regarding dosing, safety and preliminary efficacy to inform potential pediatric labeling" by designing and executing earlier rational dose finding and signal seeking trials [19]. The Alliance for Childhood Cancer, representing more than 30 national patient advocacy groups and professional medical and scientific organizations invested in advancing the interests of children with cancer, applauded the inclusion of the RACE for Children Act in the FDA Reauthorization Act of 2017 (FDARA) legislation, passed in the Senate and in the House in July of 2017.

These new regulatory initiatives by EMA and FDA combined with umbrella clinical trial initiatives aimed at allowing children and adolescents with relapsed or refractory pediatric cancers early access to promising targeted precision medicines have the potential to significantly alter the therapeutic landscape for difficult-to-treat pediatric/adolescent cancers for the benefit of current and future pediatric cancer patients [20].

\section{References}

1. AYAO (2006) Adolescent and Young Adult Oncology Progress Review Group. Report of the Adolescent and Young Adult Progress Review Group. US Department of Health and Human Services, National Institutes of Health, National Cancer Institute and LiveStrong Young Adult Alliance.

2. Bleyer A, Tai E, Siegel S (2018) Role of clinical trials in survival progress of American adolescents and young adults with cancer-and lack thereof. Pediatr Blood Cancer 65: e27074.
3. Chuk MH, Mulugeta Y, Cline MR, Mehrotra N, Reaman GH (2017) Enrolling adolescents in disease/target-appropriate adult oncology clinical trials of investigational agents. Clin Cancer Res 23: 9-12.

4. Livestrong (2006) Closing the gap: Research and care imperatives for adolescents and young adults with cancer.

5. US FDA (2018) Considerations for the Inclusion of Adolescent Patients in Adult Oncology Clinical Trials Guidance for Industry. Draft Guidance.

6. Freyer DR, Felgenhauer J, Perentesis J (2013) COG Adolescent and Young Adult Oncology Discipline Committee. Children's Oncology Group's 2013 blueprint for research: adolescent and young adult oncology. Pediatr Blood Cancer 60: 1055-1058.

7. Gaspar N, Marshall LV, Binner D (2018) Joint adolescent-adult early phase clinical trials to improve access to new drugs for adolescents with cancer: proposals from the multi-stakeholder platform-ACCELERATE. Ann Oncol 29: 766-771.

8. Kim ES, Bruinooge SS, Roberts S (2017) Broadening eligibility criteria to make clinical trials more representative: American Society of Clinical Oncology and Friends of Cancer Research Joint Research Statement. J Clin Oncol 35: 3737-3744.

9. Regulation (EC) No 1901/2006 of the European Parliament and of the Council of 12 December 2006 on medicinal products for pediatric use and amending Regulation (EEC) No 1768/92, Directive 2001/20/EC, Directive 2001/83/EC and Regulation (EC) No $726 / 2004$

10. Smith AW, Seibel NL, Lewis DR, Albritton KH, Blair DF, et al. (2016) Next steps for adolescent and young adult oncology workshop: An update on progress and recommendations for the future. Cancer 122: 988-999. [Crossref]

11. Stark D, Bielack S, Brugieres L (2016) Teenagers and young adults with cancer in Europe: from national programmes to a European integrated coordinated project. Eur $J$ Cancer Care (Engl) 25: 419-427.

12. Stefanie M. Thomas MD, Jemily Malvar MS, Henry Tran MD, Jared Shows MD, et al. (2018) A prospective, observational cohort study comparing cancer clinical trial availability and enrollment between early adolescents/young adults and children. Cancer 124: 983-990.

13. Thomas SM, Malvar J, Tran H (2018) A prospective, observational cohort study comparing cancer clinical trialavailability and enrollment between early adolescents young adults and children. Cancer 124: 983-990.

14. Vassal G, Rousseau R, Blanc P (2015) Creating a unique, multi-stakeholder Pediatric Oncology Platform to improve drug development for children and adolescents with cancer. Eur J Cancer 51: 218-224.

15. Burke ME, Albritton K, Marina N (2007) Challenges in the recruitment of adolescents and young adults to cancer clinical trials. Cancer 110: 2385-2393. [Crossref]

16. Fern LA, Taylor RM (2018) Enhancing accrual to clinical trials of adolescents and young adults with cancer. Pediatr Blood Cancer 65: e27233. [Crossref]

17. Forrest SJ, Geoerger B, Janeway KA (2018) Precision medicine in pediatric oncology. Curr Opin Pediatr 30: 17-24. [Crossref]

18. Laetsch TW, DuBois SG, Nagasubramanian R (2017) A pediatric phase I study of larotrectinib, a highly selective inhibitor of the tropomyosin receptor kinase (TRK) family. J Clin Oncol 35: 10510.

19. Reaman G (2017) ASCO Pediatric Oncology Award and Lecture, 2018 ASCO Annual Symposium; Relevant Molecular Targets in Pediatric Cancers: Applicability to Pediatric Therapeutic Investigations Required Under FDARA 2017. FDA Briefing Document, Pediatric Oncology Subcommittee of the Oncologic Drugs Advisory Committee (ODAC)

20. Beaver JA, Ison G, Pazdur R (2017) Reevaluating eligibility criteria —-balancing patient protection and participation in oncology trials. N Engl J Med 376: 1504-1505.

Copyright: (C2018 Uckun FM. This is an open-access article distributed under the terms of the Creative Commons Attribution License, which permits unrestricted use, distribution, and reproduction in any medium, provided the original author and source are credited. 